Article

\title{
Multicriteria Correlation Preference Information (MCCPI)-Based Ordinary Capacity Identification Method
}

\author{
Jian-Zhang $W_{u} * \mathbb{D}$, Yi-Ping Zhou, Li Huang and Jun-Jie Dong \\ School of Business, Ningbo University, Ningbo 315211, China; zhouypnbdx@163.com (Y.-P.Z.); \\ hlnhym11@163.com (L.H.); superd2j@163.com (J.-J.D.) \\ * Correspondence: sjzwjz@gmail.com; Tel.: +86-150-5883-6418
}

Received: 24 February 2019; Accepted: 19 March 2019; Published: 24 March 2019

check for updates

\begin{abstract}
Multicriteria correlation preference information (MCCPI) refers to a special type of 2-dimensional explicit information: the importance and interaction preferences regarding multiple dependent decision criteria. A few identification models have been established and implemented to transform the MCCPI into the most satisfactory 2-additive capacity. However, as one of the most commonly accepted particular type of capacity, 2-additive capacity only takes into account 2-order interactions and ignores the higher order interactions, which is not always reasonable in a real decision-making environment. In this paper, we generalize those identification models into ordinary capacity cases to freely represent the complicated situations of higher order interactions among multiple decision criteria. Furthermore, a MCCPI-based comprehensive decision aid algorithm is proposed to represent various kinds of dominance relationships of all decision alternatives as well as other useful decision aiding information. An illustrative example is adopted to show the proposed MCCPI-based capacity identification method and decision aid algorithm.
\end{abstract}

Keywords: multicriteria decision analysis; capacity; MCCPI; shapley importance and interaction index; dominance relationship diagram

\section{Introduction}

Capacity [1] (or fuzzy measure [2]) flexibly describes the importance and interaction among multiple decision criteria by means of evaluating the measures of all coalitions of the decision criteria [3-5]. However, this evaluation process is actually double-edged, also bringing an exponential complexity, a major obstacle for the application of capacity-based multiple criteria analysis, such as multicriteria decision making [6-8]. There are two main directions as regards overcoming this major obstacle. The first is to sensibly eliminate the coefficients of ordinary capacity to construct some meaningfully particular types of capacities, such as the $\lambda$-capacity [2], the possibility and necessity capacity [9], the $\perp$-decomposable capacity [10], the $p$-symmetric capacity [11], the $k$-additive capacity (e.g., the 2-additive capacity, the most famous one in this family) [12], the $k$-tolerant and $k$-intolerant capacities [13], and $k$-maxitive capacity $[12,14]$. The second resorts to optimization theory and tools to help the decision maker to smoothly and efficiently obtain the most desired ordinary capacities or particular families of capacities from the decision maker's explicit and implicit preference information about the multiple decision criteria according to a certain principle $[6,8,15,16]$.

On the basis of the identification principle or equally the objective function of the optimization model, the capacity identification methods can be classified into two categories $[5,17,18]$. The first category mainly constructs the objective functions by using the implicit preference of the decision maker, known as the learning set [8], which consists of some typical known alternatives and the 
decision maker's preference on them. Learning set-based methods include most of the existing capacity identification methods, such as the linear programming-based methods, e.g., the maximum split method [19], the least absolute deviation method [20], and the nonadditive robust ordinal regression (NAROR) method [21-24], and the nonlinear programming-based methods, e.g., the least-squares method [8,25], the the maximum log-likelihood and margin method [26-28], and the tool for ordinal multi-attribute sorting and ordering (TOMASO) [29]. The second category usually constructs the selection principle of the most satisfactory capacity by only using the explicit preference information, i.e., the decision maker's direct judgement about the importance and interaction of the decision criteria. The maximum entropy principle method [30-33], the compromise principle method [18], and the multicriteria correlation preference information (MCCPI)-based least deviation principle are three typical cases of the second category.

MCCPI is a kind of 2-dimensional (including both importance and interaction) pairwise preference information provided by the decision maker [5], and can be regarded as a sort of extension of the traditional 1-dimensional (only the importance) pairwise preference information, like in the Analytic Hierarchy Process (AHP) [34]. By using the refined diamond diagram or according to the 2-dimensional scale table, the decision maker can show his/her specific MCCPI [5]. On the basis of the MCCPI, some least-square- and absolute deviation principle-based identification models have been proposed to elicit the most satisfactory 2-additive capacities [5].

The 2-additive capacities, as the most famous subclass of $k$-additive capacities, have been widely accepted and used in many applications of multicriteria analysis because of their simplicity of structure [3,6]. However, this simplicity is achieved by directly cutting off the higher order interactions, i.e., 2-additive capacities assume no interaction exists among three or more decision criteria no matter what kinds and how strong the interactions of all pairs of decision criteria are. In fact, in most practical situations, we need to further take into account (or at least not neglect arbitrarily) three or higher order interactions of multiple decision criteria $[4,17,35]$. Hence, it becomes necessary to discuss how to derive the most satisfactory ordinary capacities or other particular families of capacities from MCCPI. The main aim of this paper is to generalize the 2-additive capacity identification method into the ordinary capacity identification case.

Furthermore, contrary to traditional thoughts about the most satisfactory principle, whereby only the optimal capacity is adopted to show the representative ranking order of all alternatives, we propose an MCCPI-based comprehensive decision aid method in which the whole dominance situation of all alternatives can be obtained and visualized. On the basis of the frequencies of dominance relationships, a final consistent dominance relationship or the most creditable ranking order of all alternatives can also be introduced.

This paper is organized as follows. Some preliminary knowledge about capacity, its equivalent representations, special type, and Choquet integral are introduced in Section 2. In Section 3, we briefly discuss some aid tools and corresponding rules to obtain MCCPI, and extend the MCCPI-based 2-additive capacity identification method into ordinary capacity cases. Section 4 gives an illustrative example of the two types of identification methods. Section 5 introduces a comprehensive decision aid algorithm as a further complement to the MCCPI-based capacity identification method. Finally, we conclude the paper in Section 6.

\section{Preliminaries}

Let $N=\{1,2, \ldots, n\}, n \geq 2$ be the decision criteria set, $\mathcal{P}(N)$ be its power set, and $|S|$ be the amount of elements in $S$. The definitions listed in this section can be found in References [1-4,6,9,25,36-39].

Definition 1. A set function $\mu: \mathcal{P}(N) \rightarrow[0,1]$ is called a capacity on $N$ if $\forall A, B \subseteq N, A \subseteq B$ implies $\mu(A) \leq \mu(B)$ with the boundary conditions $\mu(\varnothing)=0$ and $\mu(N)=1$. 
The number $\mu(A)$ is usually considered to be the importance of $A$. The monotonicity with respect to set inclusion given in condition (ii), with its explicit representation form, the nonadditivity with respect to disjoint subsets, enables the capacity to flexibly represent the complicated and various interaction phenomena of multiple decision criteria.

The Choquet integral, as a brilliant extension of the Lebesgue integral in a nonadditive environment, is widely accepted to aggregate the partial evaluation information on multiple interdependent decision criteria.

Definition 2. Let $x$ be a real-valued function on $N, x:=\left(x_{1}, x_{2}, \ldots, x_{n}\right) \in[0,1]^{n}$, the Choquet integral of $x$ with respect to a capacity $\mu$ on $N$ is defined as

$$
C_{\mu}(x)=\sum_{i=1}^{n}\left[x_{(i)}-x_{(i-1)}\right] \mu\left(N_{(i)}\right)
$$

or equally by

$$
C_{\mu}(x)=\sum_{i=1}^{n}\left[\mu\left(N_{(i)}\right)-\mu\left(N_{(i+1)}\right)\right] x_{(i)}
$$

where the parentheses used for indices represent a permutation on $N$ such that $x_{(1)} \leq \cdots \leq x_{(n)}, x_{(0)}=0$, $N_{(i)}=\{(i), \ldots,(n)\}$, and $N_{(n+1)}=\varnothing$.

The importance of the decision criteria and interaction among them can be measured by Shapley importance and interaction index, which has many good axiomatic characteristics suitable for decision making.

Definition 3. Let $\mu$ be a capacity on $N$, the Shapley importance and interaction index of any subset $A \subseteq N$ with respect to $\mu$ is defined as

$$
I_{\mu}(A)=\sum_{B \subseteq N \backslash A} \frac{1}{(|N|-|A|+1)}\left(\begin{array}{c}
|N|-|A| \\
|B|
\end{array}\right)^{-1}\left(\sum_{C \subseteq A}(-1)^{|A \backslash C|} \mu(C \cup B)\right) .
$$

It is generally accepted and widely used in the existing references about capacity-based decision making that the overall importance of criterion $i \in N$ is described by $I_{\mu}(i)$ and the interaction of the criteria in subset $A,|A| \geq 2$, by $I_{\mu}(A)$.

Another important transformation of capacity is the Möbius representation. It is efficient in presenting the capacity and Choquet integral, as well as in constructing capacity identification methods, and hence successfully provides another systematic representation approach of the capacity related theory.

Definition 4. Let $\mu$ be a capacity on $N$, the Möbius representation of any subset $A \subseteq N$ with respect to $\mu$ is defined as

$$
m_{\mu}(A)=\sum_{B \subseteq A}(-1)^{|A \backslash B|} \mu(B) .
$$

In fact, any set function $\mu: \mathcal{P}(N) \rightarrow \mathcal{R}, \mathcal{R}=(-\infty,+\infty)$ can be uniquely expressed in terms of its Möbius representation by

$$
\mu(A)=\sum_{B \subseteq A} m_{\mu}(B), \forall A \subseteq N .
$$


A set of $2^{n}$ coefficients $\left\{m_{\mu}(A)\right\}_{A \subseteq N}$ corresponds to the Möbius representation of a capacity on $N$ if and only if

$$
\left\{\begin{array}{l}
m_{\mu}(\varnothing)=0, \quad \sum_{A \subseteq N} m_{\mu}(A)=1, \\
\sum_{B \subseteq A, i \in B} m_{\mu}(B) \geq 0, \forall A \subseteq N, \forall i \in A .
\end{array}\right.
$$

The Choquet integral of $x:=\left(x_{1}, x_{2}, \ldots, x_{n}\right)$ with respect to the capacity $\mu$ can also be rewritten in terms of Möbius representation as

$$
C_{m_{\mu}}(x)=\sum_{A \subseteq N} m_{\mu}(A) \bigwedge_{i \in A} x_{i}
$$

In terms of the Möbius representation, the Shapley interaction index $I_{\mu}(A)$ can be rewritten as

$$
I_{m_{\mu}}(A)=\sum_{B \subseteq N \backslash A} \frac{1}{|B|+1} m_{\mu}(A \cup B) .
$$

Even the well-known k-additive capacity is also constructed in terms of the Möbius representation.

Definition 5. Let $k \in\{1,2, \ldots, n\}$, a capacity $\mu$ is said to be $k$-additive if its Möbius representation satisfies $m_{\mu}(A)=0$ for all $A \subseteq N$ such that $|A|>k$ and there exists at least one subset $A$ of $k$ elements such that $m_{\mu}(A) \neq 0$.

A 2-additive capacity only takes into account the interaction between every pair of criteria and ignores higher order interactions. This simplicity reduces the cost of constructing capacity as well as the expense of the calculating identification method, but simultaneously limits the representation ability of higher order interactions among the decision criteria to a drastic extent.

\section{MCCPI-Based 2-Additive and Ordinary Capacity Identification Method}

The MCCPI can be obtained by the refined diamond diagram as well as the 2-dimensional scale table [5].

The refined diamond diagram is shown in Figure 1, where line segment OA or the interval $[0,1]$ is divided into eight equal portions to represent the following nine categories of the relative importance cases between two given decision criteria: the criterion $i$ is extremely less (resp. very strongly less, strongly less, slightly less, equally, slightly more, strongly more, very strongly more, and extremely more) important than the criterion $j$ if and only if the first coordinate of the point belongs to $[0.00,0.125)(\operatorname{resp}[0.125,0.25),[0.25,0.375),[0.375,0.50), 0.50,(0.50,0.625],(0.625,0.75],(0.75,0.875]$, and $(0.875,1.00])$. For convenience, we can call these intervals the relative importance intervals.

The partial interaction cases between criteria can also be divided into the following nine categories (see Figure 1): The almost zero (resp. slightly positive, strongly positive, very strongly positive, extremely positive, slightly negative, strongly negative, very strongly negative, and extremely negative) corresponds to the points in the sub-diamond $\mathrm{OBAB}^{\prime}$ (resp. OBAC, OCAD , ODAE , OEAF , $\mathrm{OB}^{\prime} \mathrm{AC}^{\prime}$, $\mathrm{OC}^{\prime} \mathrm{AD}^{\prime}, \mathrm{OD}^{\prime} \mathrm{AE}^{\prime}$, and $\left.\mathrm{OE}^{\prime} \mathrm{AF}^{\prime}\right)$, where the points $\mathrm{O}=(0.00,0.00), \mathrm{A}=(1.00,0.00), \mathrm{B}=(0.50,0.05)$ ${ }^{\prime} \mathrm{B}^{\prime}=(0.50,-0.05), \mathrm{C}=(0.50,0.25), \mathrm{E}=(0.50,0.75), \mathrm{F}=(0.50,1.00), \mathrm{C}^{\prime}=(0.50,-0.25)$, $\mathrm{D}^{\prime}=(0.50,-0.50), \mathrm{E}^{\prime}=(0.50,-0.75)$, and $\mathrm{F}^{\prime}=(0.50,-1.00)$. Basically, the partial interaction intervals of the above nine categories are the multiplication of the minimum of two relative importance values with the corresponding ratio intervals, which are $[-0.05,0.05],[0.05,0.5],[0.5,1],[1,1.5],[1.5,2]$, $[-0.5,-0.05],[-1,-0.5],[-1.5,-1]$, and $[-2,-1.5]$. 


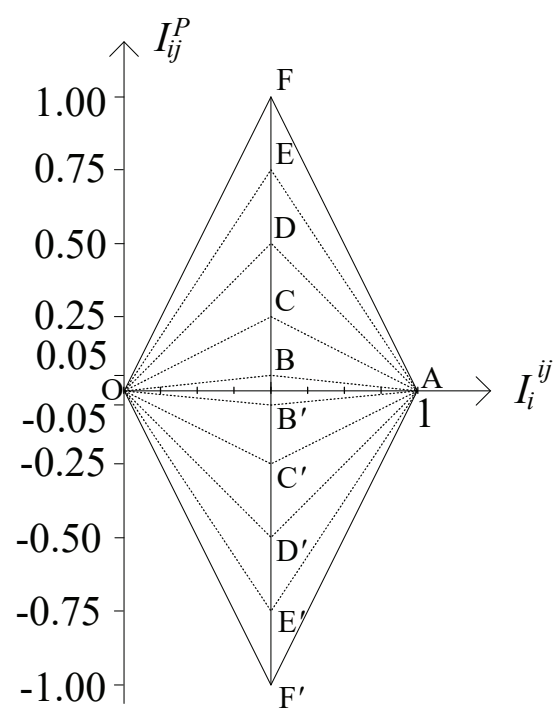

Figure 1. Refined diamond diagram.

On the basis of the above rules, the decision maker can roughly estimate a point in the above $9 \times 9=81$ refined segments in the diamond to specifically represent the MCCPI, the relative importance and partial interaction value of an arbitrary given pair of criteria [5]. It is undoubted that different points, even in the same refined segment, may generate different optimal Shapley importance and interaction values, and may eventually generate different overall evaluations and ranking order of the decision alternatives. In order to unify this subjective judgement information just like the 1-9 scale of preference judgment in the AHP, we can choose the medians of the 81 refined segments as representatives of MCCPI, see Table 1.

Table 1. The scale of 2-dimensional multicriteria correlation preference information (MCCPI).

\begin{tabular}{cc}
\hline \multicolumn{2}{c}{ Relative Importance of Criterion $i$ to $j$} \\
\hline Nine Categories & Scale Value of \\
\hline extremely less important & 0.0625 \\
very strongly less important & 0.1875 \\
strongly less important & 0.3125 \\
slightly less important & 0.4375 \\
equally important & 0.5000 \\
slightly more important & 0.5625 \\
strongly more important & 0.6875 \\
very strongly more important & 0.8125 \\
extremely more important & 0.9375 \\
\hline Partial Interaction between $i$ and $j$ \\
\hline Nine Categories & Scale Value of \\
\hline extremely positive & $1.75 \min \left(I_{i}^{i j}, 1-I_{i j}^{i j}\right)$ \\
very strongly positive & $1.25 \min \left(I_{i}^{i j}, 1-I_{i}^{i j}\right)$ \\
strongly positive & $0.75 \min \left(I_{i}^{i j}, 1-I_{i}^{i j}\right)$ \\
slightly positive & $0.3 \min \left(I_{i}^{i j}, 1-I_{i}^{i j}\right)$ \\
almost zero & 0.000 \\
slightly negative & $-0.3 \min \left(I_{i}^{i j}, 1-I_{i}^{i j}\right)$ \\
strongly negative & $-0.75 \min \left(I_{i,}^{i j}, 1-I_{i}^{i j}\right)$ \\
very strongly negative & $-1.25 \min \left(I_{i}^{i j}, 1-I_{i}^{i j}\right)$ \\
extremely negative & $-1.75 \min \left(I_{i}^{i j}, 1-I_{i}^{i j}\right)$ \\
\hline
\end{tabular}


We can collect the relative importance and partial interaction obtained from the $\left(\begin{array}{c}n \\ 2\end{array}\right)$ times 2-dimensional pairwise comparisons into two matrices:

$$
\mathbf{R}=\left[r_{i j}\right]_{n \times n}=\left[I_{i}^{i j}\right]_{n \times n}
$$

and

$$
\mathbf{P}=\left[p_{i j}\right]_{n \times n}=\left[I_{i j}^{P}\right]_{n \times n},
$$

where $I_{i}^{i j}$ is the relative importance value of criterion $i$ to criterion $j, I_{i j}^{P}$ is the partial interaction value between criteria $i$ and $j$. Since $I_{i}^{i j}+I_{j}^{i j}=1, I_{i j}^{P}=I_{j i}^{P}$, we have $r_{i j}+r_{j i}=1, p_{i j}=p_{j i}$. For the sake of consistency, we can set $r_{i i}=1 / 2, i=1,2, \ldots, n$.

Let $\mu$ be one of the most satisfactory 2 -additive capacities for the decision maker. If the MCCPI is consistent and totally caters to the importance and interaction preference associated with $\mu$, we have the following statements [5]:

$$
\begin{gathered}
r_{i j}=I_{i}^{i j}=\frac{I_{\mu}(i)}{I_{\mu}(i)+I_{\mu}(j)} \\
p_{i j}=I_{i j}^{P}=\frac{I_{\mu}(\{i, j\})}{I_{\mu}(i)+I_{\mu}(j)} .
\end{gathered}
$$

Obviously, the above equation show an ideal situation. In reality, some deviation exists more or less between the left- and right-hand sides. However, we can find out the minimum gap between the two sides with the help of optimization models. By introducing the distance measure and the boundary and monotonicity conditions, the 2-additive capacity identification model can be constructed as [5]:

$$
\min z=\sum_{i, j \in N, i \neq j}\left(D\left(r_{i j}, \frac{I_{\mu}(i)}{I_{\mu}(i)+I_{\mu}(j)}\right)+D\left(p_{i j}, \frac{I_{\mu}(\{i, j\})}{I_{\mu}(i)+I_{\mu}(j)}\right)\right)
$$

s.t. Boundary and monotonicity conditions of 2-additive capacity,

where the distance can be the quadratic or absolute form and the objective function aims to minimize the divergence between the left and right sides in Equations (1) and (2).

As one special class of $k$-additive capacities, 2-additive capacities only take into account the interaction between every pair of criteria and ignores the higher order interactions [3]. However, in most practical situations, we need to consider the higher order interactions among decision criteria (i.e., the interaction among three or more decision criteria), although it is relative difficult for the decision maker to identify the exact value or degree of the high order interactions; at least we can not just set those higher order interactions to zero in any situation. Hence, it is necessary to extend the above model into the ordinary capacity identification model. This task can be easily done by changing the conditions of 2-additive capacity into the conditions of ordinary capacity (as shown in Definition 1). We can also include some additional preference information from the decision maker on higher order subsets' importance and interaction to enhance the model's representation ability. Now, we can represent the ordinary capacity identification model as follows:

$$
\min z=\sum_{i, j \in N, i \neq j}\left(D\left(r_{i j}, \frac{I_{\mu}(i)}{I_{\mu}(i)+I_{\mu}(j)}\right)+D\left(p_{i j}, \frac{I_{\mu}(\{i, j\})}{I_{\mu}(i)+I_{\mu}(j)}\right)\right)
$$

s.t. Boundary and monotonicity conditions of the capacity,

Preference on higher order subsets' importance and interaction. 
In model (4), the capacity can be ordinary or any particular type of capacity, and the higher order preference can be given in comparison or interval form as has been shown in other works $[5,8,24,32,40,41]$. In the following, we illustrate the methods using an example.

\section{Empirical Analysis Using an Example}

See the application example in Reference [5]: a decision maker has to rank seven cars according to four criteria: price in Yuan, acceleration $0-100 \mathrm{~km} / \mathrm{h}$ in seconds, maximum speed in $\mathrm{km} / \mathrm{h}$, and consumption in $\mathrm{km} / \mathrm{L}$, denoted as criteria $1,2,3$, and 4 . These cars' partial evaluations on four criteria are given in Table 2.

Table 2. The partial evaluations of the seven types of cars.

\begin{tabular}{cccccccc}
\hline & Car A & Car B & Car C & Car D & Car E & Car F & Car G \\
\hline Price & 0.153 & 0.256 & 0.823 & 0.939 & 0.675 & 0.876 & 0.922 \\
Acceleration & 0.586 & 0.902 & 0.557 & 0.721 & 0.689 & 0.354 & 0.687 \\
Max speed & 0.483 & 0.854 & 0.493 & 0.638 & 0.472 & 0.277 & 0.748 \\
Consumption & 0.845 & 0.417 & 0.521 & 0.712 & 0.611 & 0.758 & 0.691 \\
\hline
\end{tabular}

The decision maker's MCCPI is given as follows:

- Criterion 1 is strongly more important than 2 and their partial interaction is extremely positive;

- Criterion 1 is strongly more important than 3 and their partial interaction is strongly positive;

- Criterion 1 is slightly less important than 4 and their partial interaction is very strongly negative;

- Criterion 2 is strongly more important than 3 and their partial interaction is slightly negative;

- Criterion 2 is slightly less important than 4 and their partial interaction is strongly positive;

- Criterion 3 is strongly less important than 4 and their partial interaction is strongly positive.

Using the above six 2-dimensional pairwise comparisons, the decision maker's above MCCPI can be specifically represented in Figure 2.

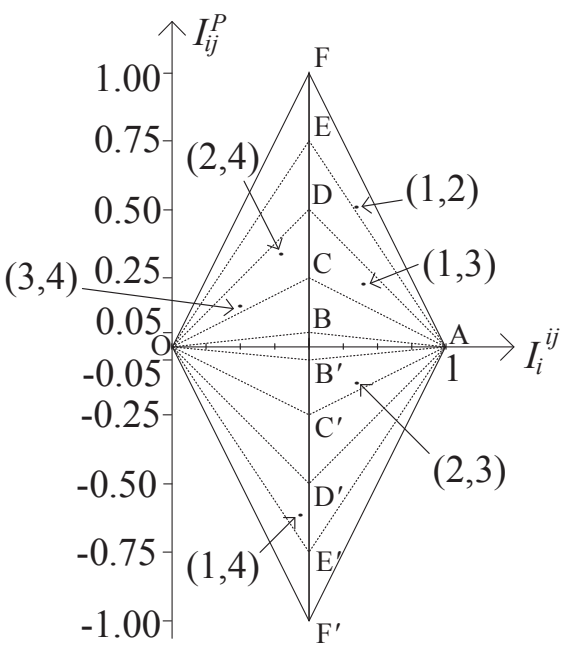

Figure 2. MCCPI of the four criteria.

In terms of matrix form, we get

$$
\mathbf{R}=\left[\begin{array}{llll}
0.500 & 0.667 & 0.700 & 0.471 \\
0.333 & 0.500 & 0.667 & 0.400 \\
0.300 & 0.333 & 0.500 & 0.250 \\
0.429 & 0.600 & 0.750 & 0.500
\end{array}\right]
$$


and

$$
\mathbf{P}=\left[\begin{array}{cccc}
- & 0.550 & 0.230 & -0.610 \\
0.550 & - & -0.130 & 0.340 \\
0.230 & -0.130 & - & 0.150 \\
-0.610 & 0.340 & 0.150 & -
\end{array}\right]
$$

We can introduce the absolute distance and construct models according to (3) and (4) to obtain the optimal 2-additive and ordinary capacity, respectively. For example, the ordinary capacity identification model in terms of Möbius representation can be given as follows:

$$
\begin{gathered}
\min z=\sum_{i=1}^{3} \sum_{j>i}^{4}\left(d_{i j}^{-}+d_{i j}^{+}+e_{i j}^{-}+e_{i j}^{+}\right) \\
\left\{\begin{array}{c}
m_{\mu}(\varnothing)=0, \quad m_{\mu}(\{1\})+\cdots+m_{\mu}(\{1,2,3,4\})=1, \\
m_{\mu}(\{1\}) \geq 0, m_{\mu}(\{2\}) \geq 0, \\
\vdots \\
m_{\mu}(\{4\})+\cdots+m_{\mu}(\{1,2,3,4\}) \geq 0, \\
0.667\left(I_{m_{\mu}}(1)+I_{m_{\mu}}(2)\right)-I_{m_{\mu}}(1)+d_{12}^{-}-d_{12}^{+}=0, \\
0.700\left(I_{m_{\mu}}(1)+I_{m_{\mu}}(3)\right)-I_{m_{\mu}}(1)+d_{13}^{-}-d_{13}^{+}=0, \\
\vdots \\
0.250\left(I_{m_{\mu}}(3)+I_{m_{\mu}}(4)\right)-I_{m_{\mu}}(3)+d_{34}^{-}-d_{34}^{+}=0, \\
0.550\left(I_{m_{\mu}}(1)+I_{m_{\mu}}(2)\right)-I_{m_{\mu}}(\{1,2\})+e_{12}^{-}-e_{12}^{+}=0, \\
0.230\left(I_{m_{\mu}}(1)+I_{m_{\mu}}(3)\right)-I_{m_{\mu}}(\{1,3\})+e_{13}^{-}-e_{13}^{+}=0, \\
\vdots \\
0.150\left(I_{m_{\mu}}(3)+I_{m_{\mu}}(4)\right)-I_{m_{\mu}}(\{3,4\})+e_{34}^{-}-e_{34}^{+}=0,
\end{array}\right.
\end{gathered}
$$

where the deviation variables $d_{i j}^{-}, d_{i j}^{+}, e_{i j}^{-}, e_{i j}^{+} \geq 0, d_{i j}^{-} \times d_{i j}^{+}=0, e_{i j}^{-} \times e_{i j}^{+}=0, i, j \in\{1,2,3,4\}, i<j$, are introduced to transform the absolute distance, the nonlinear representations, into linear constraints. The Möbius representation and Shapely interaction index of the optimal capacities are given in Table 3 .

Table 3. The interaction indices of the optimal capacities.

\begin{tabular}{cccccc}
\hline $\begin{array}{c}\text { Möbius } \\
\text { Representation }\end{array}$ & $\begin{array}{c}\text { 2-Additive } \\
\text { Capacity }\end{array}$ & $\begin{array}{c}\text { Ordinary } \\
\text { Capacity }\end{array}$ & $\begin{array}{c}\text { Shapley Interaction } \\
\text { Index }\end{array}$ & $\begin{array}{c}\text { 2-Additive } \\
\text { Capacity }\end{array}$ & $\begin{array}{c}\text { Ordinary } \\
\text { Capacity }\end{array}$ \\
\hline$m_{\mu}(\varnothing)$ & 0 & 0 & $I_{\mu}(\varnothing)$ & 0.4802 & 0.4770 \\
$m_{\mu}(\{1\})$ & 0.3980 & 0.2763 & $I_{\mu}(\{1\})$ & 0.3545 & 0.3081 \\
$m_{\mu}(\{2\})$ & 0.0420 & 0.0000 & $I_{\mu}(\{2\})$ & 0.2150 & 0.2307 \\
$m_{\mu}(\{3\})$ & 0.0430 & 0.0539 & $I_{\mu}(\{3\})$ & 0.1075 & 0.1153 \\
$m_{\mu}(\{4\})$ & 0.3980 & 0.4137 & $I_{\mu}(\{4\})$ & 0.3230 & 0.3459 \\
$m_{\mu}(\{1,2\})$ & 0.2050 & 0.4437 & $I_{\mu}(\{1,2\})$ & 0.2050 & 0.2941 \\
$m_{\mu}(\{1,3\})$ & 0.1060 & 0.2247 & $I_{\mu}(\{1,3\})$ & 0.1060 & 0.0974 \\
$m_{\mu}(\{1,4\})$ & -0.3980 & -0.2763 & $I_{\mu}(\{1,4\})$ & -0.3980 & -0.3990 \\
$m_{\mu}(\{2,3\})$ & -0.0420 & 0.0000 & $I_{\mu}(\{2,3\})$ & -0.0420 & -0.0450 \\
$m_{\mu}(\{2,4\})$ & 0.1830 & 0.2364 & $I_{\mu}(\{2,4\})$ & 0.1830 & 0.1961 \\
$m_{\mu}(\{3,4\})$ & 0.0650 & 0.0872 & $I_{\mu}(\{3,4\})$ & 0.0650 & 0.0692 \\
$m_{\mu}(\{1,2,3\})$ & 0 & -0.2786 & $I_{\mu}(\{1,2,3\})$ & 0 & -0.0921 \\
$m_{\mu}(\{1,2,4\})$ & 0 & -0.2693 & $I_{\mu}(\{1,2,4\})$ & 0 & -0.0828 \\
$m_{\mu}(\{1,3,4\})$ & 0 & -0.2247 & $I_{\mu}(\{1,3,4\})$ & 0 & -0.0382 \\
$m_{\mu}(\{2,3,4\})$ & 0 & -0.0600 & $I_{\mu}(\{2,3,4\})$ & 0 & 0.1265 \\
$m_{\mu}(\{1,2,3,4\})$ & 0 & 0.3730 & $I_{\mu}(\{1,2,3,4\})$ & 0 & 0.3730 \\
\hline
\end{tabular}


From Table 3, we can see that the 3- and 4-order internal (the Möbius representation) and comprehensive (Shapley) interaction indices can be distinguished as being non-zero: see the last five rows in third and sixth columns. This means some meaningful interaction information could be cut out if the interaction phenomenon of these four criteria was just set as a 2-order situation.

It should be pointed out that the matrices $\mathbf{R}$ and $\mathbf{P}$ or equally the points in Figure 2 are just one special case of the decision maker's MCCPI about the four criteria, see Table 4. If one adopts the MCCPI in last column, which is obtained according to the scale table, see Table 1, we can get different, though not very different, optimal 2-additive and ordinary capacities, and then achieve the different overall evaluations and ranking order of the seven cars, as shown in Table 5. One can see that in both cases of MCCPI, by the points in Figure 2 and the scales values, the ranking orders obtained by ordinary capacities are quite different to those by 2-additive capacities. This means, to some degree, the 2-additive capacity achieves simplicity and convenience by sacrificing a certain amount of decision information.

Table 4. The MCCPI and representative values.

\begin{tabular}{cccc}
\hline Criteria & MCCPI & Point in Figure 2 & Scale Values \\
\hline 1 vs. 2 & (strongly more, extremely positive) & $(0.667,0.550)$ & $(0.6875,0.5467)$ \\
1 vs. 3 & (strongly more, strongly positive) & $(0.700,0.230)$ & $(0.6875,0.2343)$ \\
1 vs. 4 & (slightly less, strongly negative) & $(0.471,-0.610)$ & $(0.4375,-0.5469)$ \\
2 vs. 3 & (strongly more, slightly negative) & $(0.667,-0.130)$ & $(0.6875,-0.0938)$ \\
2 vs. 4 & (slightly less, strongly positive) & $(0.400,0.340)$ & $(0.4375,0.3281)$ \\
3 vs. 4 & (strongly less, strongly positive) & $(0.250,0.150)$ & $(0.3125,0.2344)$ \\
\hline
\end{tabular}

Table 5. The overall evaluations and ranking orders of the seven types of cars.

\begin{tabular}{ccccccccccc}
\hline \multirow{2}{*}{ MCCPI } & $\begin{array}{c}\text { Type of } \\
\text { Capacity }\end{array}$ & & Car A & Car B & Car C & Car D & Car E & Car F & Car G \\
& \multirow{2}{*}{ 2-additive } & evaluations & 0.5476 & 0.3877 & 0.6453 & 0.7918 & 0.6290 & 0.5485 & 0.7899 \\
Points in Figure 2 & & ranking & 6 & 7 & 3 & 1 & 4 & 5 & 2 \\
& ovaluations & 0.5684 & 0.3973 & 0.6155 & 0.7657 & 0.6327 & 0.5402 & 0.7689 \\
& & ordinary & ranking & 5 & 7 & 4 & 2 & 3 & 6 & 1 \\
\hline \multirow{3}{*}{ Scale values } & \multirow{2}{*}{ 2-additive } & evaluations & 0.4990 & 0.3798 & 0.6210 & 0.7702 & 0.6191 & 0.5026 & 0.7716 \\
& & ranking & 6 & 7 & 3 & 2 & 4 & 5 & 1 \\
& ordinary & evaluations & 0.5321 & 0.3742 & 0.5922 & 0.7516 & 0.6205 & 0.4786 & 0.7442 \\
& & ranking & 5 & 7 & 4 & 1 & 3 & 6 & 2 \\
\hline
\end{tabular}

\section{Further Discussions}

As mentioned above, different representative points of the given MCCPI may lead to different overall evaluations and ranking orders of decision alternatives. How to get the whole view of the dominance situation of the decision alternatives becomes a meaningful topic inevitably encountered in practice. Considering the complicity of the capacity identification method and the computing feasibility, we give a MCCPI-based comprehensive decision aid algorithm to solve this issue, see Algorithm 1.

Remark 1. Usually, in the dominance relationship diagram obtained by Algorithm 1, for each pair of alternatives, if we choose the largest frequency dominance relationship and remove other lower chance dominance relationships, we can construct a wholly consistent dominance diagram of all alternatives, which can serve as a representative ranking order. However, it should be pointed out that some conflicts can occur among these chosen relationships.

Now, let's see again the example in Section 4. We execute Algorithm 1 with $K=100$. Table 6 shows 10 rows of matrix $\mathbf{O}$, which suggests the overall ranking orders of seven cars change mainly in 
relation to the orders of car A and car F with fifth and sixth, the orders of car $\mathrm{C}$ and car $\mathrm{E}$ with third and fourth, and the orders of car D and car G with first and second.

On the basis of the results of matrix $\mathbf{E}$ or $\mathbf{O}$, we can get the comprehensive dominance relationship diagram of the seven cars, see Figure 3.

The frequencies of most dominance relationships are 1, except that of $d^{\succ}(A, F)=0.45, d^{\prec}(A, F)=$ $0.55, d^{\succ}(C, E)=0.03, d^{\prec}(C, E)=0.97, d^{\succ}(D, G)=0.76, d^{\prec}(A, F)=0.24$. In order to verify the stability of the frequencies, we try to increase $\mathcal{K}$ to 300 and 500 , and the above six frequencies are accordingly changed to: $0.42,0.58,0.03,0.97,0.74$, and 0.26 with $\mathcal{K}=300 ; 0.36,0.64,0.04,0.96,0.73$, and 0.26 with $\mathcal{K}=500$, which means Figure 3 is highly creditable. For each pair of criteria, we eliminate the lower frequency relationship and then get the following consistent dominance relationship:

$$
\mathrm{Car} \mathrm{D} \succ \mathrm{Car} \mathrm{G} \succ \mathrm{Car} \mathrm{C} \succ \mathrm{Car} \mathrm{E} \succ \mathrm{Car} \mathrm{A} \succ \mathrm{Car} \mathrm{F} \succ \mathrm{Car} \mathrm{B} .
$$

Finally, in order to further emphasize the advantages of ordinary capacity over 2-additive capacity in flexibly when describing the higher orders' interaction situation, we provide a box plot of the Shapley interaction indices of the optimal capacities obtained with $\mathcal{K}=500$, see Figure 4, where e.g., $c(1,2,3)$ represents criteria coalition of 1,2 , and 3 . We can conclude that the 3 - and 4-order interactions are significantly non-zero.

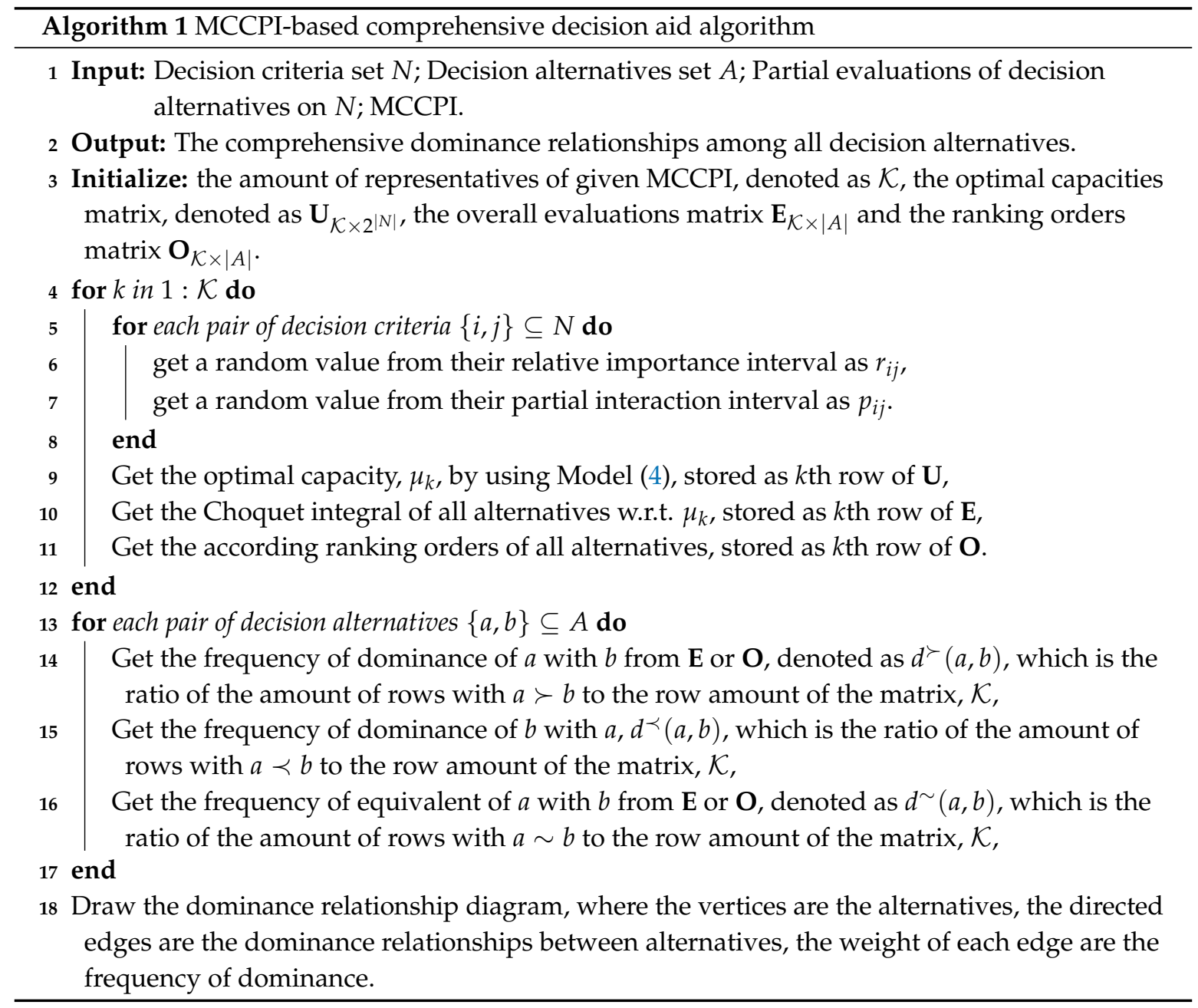


Table 6. Some ranking orders of the seven types of cars with $K=100$.

\begin{tabular}{ccccccc}
\hline Car A & Car B & Car C & Car D & Car E & Car F & Car G \\
\hline 5 & 7 & 4 & 2 & 3 & 6 & 1 \\
6 & 7 & 4 & 1 & 3 & 5 & 2 \\
6 & 7 & 3 & 1 & 4 & 5 & 2 \\
5 & 7 & 4 & 1 & 3 & 6 & 2 \\
6 & 7 & 4 & 1 & 3 & 5 & 2 \\
6 & 7 & 4 & 1 & 3 & 5 & 2 \\
6 & 7 & 4 & 2 & 3 & 5 & 1 \\
5 & 7 & 4 & 1 & 3 & 6 & 2 \\
5 & 7 & 4 & 1 & 3 & 6 & 2 \\
6 & 7 & 4 & 1 & 3 & 5 & 2 \\
\hline
\end{tabular}

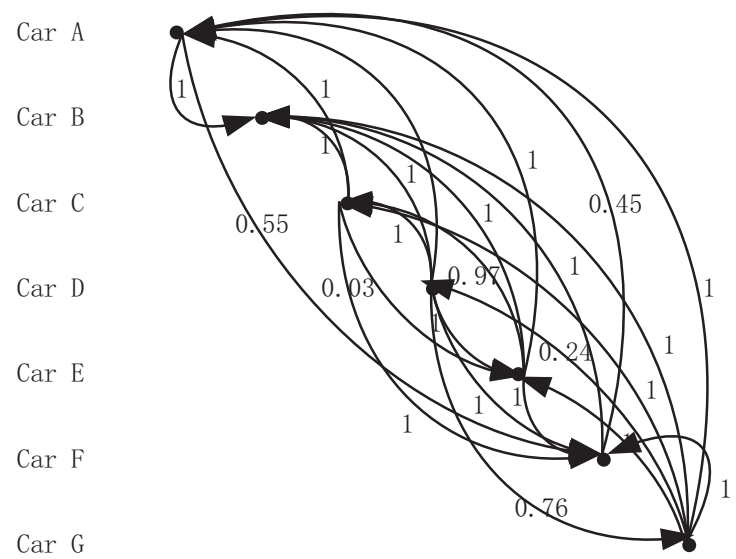

Figure 3. Dominance relationship diagram of seven cars.

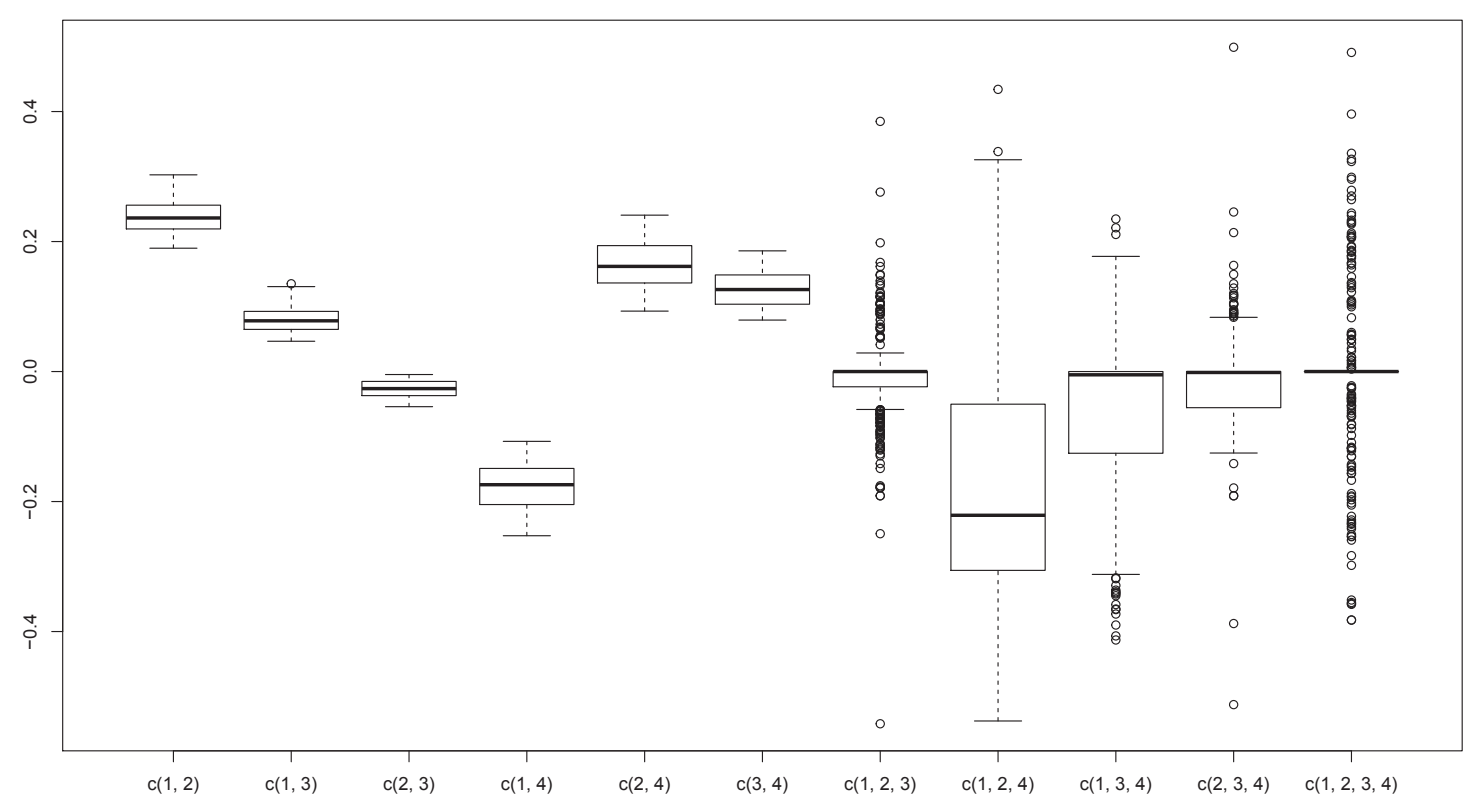

Figure 4. The box plot of Shapley indices of non-singletons with $\mathcal{K}=500$.

\section{Conclusions}

In this paper, we extend the MCCPI-based 2-additive capacity identification method to the ordinary or arbitrary particular type of capacity case. On the basis of the same 2-dimensional preference information, the new models can achieve a more flexible representation of higher interaction 
phenomena among multiple decision criteria. The MCCPI-based comprehensive decision aid algorithm can help to obtain the whole dominance relationship of all decision alternatives as well as the final comprehensive ranking order. Further research will focus on similar ways and tools for acquiring the higher order preference information, the consistency check of MCCPI, as well as the MCCPI-based multiple criteria decision aid and support software package.

Author Contributions: Methodology, J.-Z.W.; Software, J.-Z.W. and L.H.; Formal Analysis, Y.-P.Z.; Investigation, J.-J.D.; Writing_-Original Draft Preparation, J.-Z.W. and Y.-P.Z.; Writing—Review \& Editing, J.-Z.W. and J.-J.D.; Visualization, L.H.

Funding: This research was funded by the National Natural Science Foundation of China (No. 71671096) and the K.C.Wong Magna Fund in Ningbo University.

Acknowledgments: The author is grateful to the anonymous reviewers for their valuable comments and suggestions which have led to significant improvement of this paper.

Conflicts of Interest: The authors declare no conflict of interest.

\section{References}

1. Choquet, G. Theory of capacities. Ann. De L'institut Fourier 1954, 5, 131-295. [CrossRef]

2. Sugeno, M. Theory of Fuzzy Integrals and Its Applications. Ph.D. Thesis, Tokyo Institute of Technology, Tokyo, Japan, 1974.

3. Grabisch, M. k-order additive discrete fuzzy measures and their representation. Fuzzy Sets Syst. 1997, 92, 167-189. [CrossRef]

4. Beliakov, G.; James, S.; Wu, J.Z. Discrete Fuzzy Measures: Computational Aspects; Springer: Berlin, Germany, 2019.

5. Wu, J.Z.; Yang, S.; Zhang, Q.; Ding, S. 2-additive capacity identification methods from multicriteria correlation preference information. IEEE Trans. Fuzzy Syst. 2015, 23, 2094-2106. [CrossRef]

6. Beliakov, G.; Sola, H.B.; Calvo, T. A Practical Guide to Averaging Functions; Springer: New York, NY, USA, 2016.

7. Wu, J.Z.; Beliakov, G. Probabilistic bipartition interaction index of multiple decision criteria associated with the nonadditivity of fuzzy measures. Int. J. Intell. Syst. 2018, 34, 247-270. [CrossRef]

8. Grabisch, M.; Kojadinovic, I.; Meyer, P. A review of methods for capacity identification in Choquet integral based multi-attribute utility theory: Applications of the Kappalab R package. Eur. J. Oper. Res. 2008, 186, 766-785. [CrossRef]

9. Weber, R.J. Probabilistic values for games. In The Shapley Value. Essays in Honor of Lloyd S. Shapley; Roth, A., Ed.; Cambridge University Press: Cambridge, UK, 1988; pp. 101-119.

10. Weber, S. $\perp$-decomposable measures and integrals for Archimedean t-conorms $\perp$. J. Math. Anal. Appl. 1984, 101, 114-138. [CrossRef]

11. Miranda, P.; Grabisch, M.; Gil, P. p-Symmetric fuzzy measures. Int. J. Uncertain. Fuzziness Knowl. Based Syst. 2002, 10, 105-123. [CrossRef]

12. Mesiar, R. k-order additive fuzzy measures. Int. J. Uncertain. Fuzziness Knowl. Based Syst. 1999, 7, 561-568. [CrossRef]

13. Marichal, J.L. k-intolerant capacities and Choquet integrals. Eur. J. Oper. Res. 2007, 177, 1453-1468. [CrossRef]

14. Calvo, T.; De Baets, B. Aggregation operators defined by k-order additive/maxitive fuzzy measures. Int. J. Uncertain. Fuzziness Knowl. Based Syst. 1998, 6, 533-550. [CrossRef]

15. Wu, J.Z.; Yu, L.P.; Li, G.; Jin, J.; Du, B. The sum interaction indices of some particular families of monotone measures. J. Intell. Fuzzy Syst. 2016, 31, 1447-1457. [CrossRef]

16. Wu, J.Z.; Yu, L.P.; Li, G.; Jin, J.; Du, B. Using the monotone measure sum to enrich the measurement of the interaction of multiple decision criteria. J. Intell. Fuzzy Syst. 2016, 30, 2529-2539. [CrossRef]

17. Wu, J.Z.; Pap, E.; Szakal, A. Two kinds of explicit preference information oriented capacity identification methods in the context of multicriteria decision analysis. Int. Trans. Oper. Res. 2018, 25, 807-830. [CrossRef]

18. Wu, J.Z.; Zhang, Q.; Du, Q.; Dong, Z. Compromise principle based methods of identifying capacities in the framework of multicriteria decision analysis. Fuzzy Sets Syst. 2014, 246, 91-106. [CrossRef] 
19. Marichal, J.L.; Roubens, M. Determination of weights of interacting criteria from a reference set. Eur. J. Oper. Res. 2000, 124, 641-650. [CrossRef]

20. Beliakov, G. Construction of aggregation functions from data using linear programming. Fuzzy Sets Syst. 2009, 160, 65-75. [CrossRef]

21. Angilella, S.; Bottero, M.; Corrente, S.; Ferretti, V.; Greco, S.; Lami, I.M. Non Additive Robust Ordinal Regression for urban and territorial planning: An application for siting an urban waste landfill. Ann. Oper. Res. 2016, 245, 427-456. [CrossRef]

22. Angilella, S.; Greco, S.; Matarazzo, B. Non-additive robust ordinal regression: A multiple criteria decision model based on the Choquet integral. Eur. J. Oper. Res. 2010, 201, 277-288. [CrossRef]

23. Corrente, S.; Greco, S.; Ishizaka, A. Combining analytical hierarchy process and Choquet integral within non-additive robust ordinal regression. Omega 2016, 61, 2-18. [CrossRef]

24. Corrente, S.; Greco, S.; Kadziński, M.; Słowiński, R. Robust ordinal regression in preference learning and ranking. Mach. Learn. 2013, 93, 381-422. [CrossRef]

25. Grabisch, M.; Labreuche, C. A decade of application of the Choquet and Sugeno integrals in multi-criteria decision aid. Ann. Oper. Res. 2010, 175, 247-286. [CrossRef]

26. Fallah Tehrani, A.; Cheng, W.; Dembczynski, K.; Hüllermeier, E. Learning monotone nonlinear models using the Choquet integral. Mach. Learn. 2012, 89, 183-211. [CrossRef]

27. Hüllermeier, E.; Tehrani, A.F. Efficient Learning of Classifiers Based on the 2-Additive Choquet Integral. In Computational Intelligence in Intelligent Data Analysis; Moewes C., Nürnberger A., Eds.; Springer: Berlin/Heidelberg, Germany, 2013; pp. 17-29.

28. Fallah Tehrani, A.; Cheng, W.; Hüllermeier, E. Preference Learning using the Choquet Integral: The Case of Multipartite Ranking. IEEE Trans. Fuzzy Syst. 2012, 20, 1102-1113. [CrossRef]

29. Meyer, P.; Roubens, M. Choice, Ranking and Sorting in Fuzzy Multiple Criteria Decision Aid. In Multiple Criteria Decision Analysis: State of the Art Surveys; Figueira, J., Greco, S., Ehrogott, M., Eds.; Springer: New York, NY, USA, 2005; pp. 471-503.

30. Kojadinovic, I. Minimum variance capacity identification. Eur. J. Oper. Res. 2007, 177, 498-514. [CrossRef]

31. Kojadinovic, I. An axiomatic approach to the measurement of the amount of interaction among criteria or players. Fuzzy Sets Syst. 2005, 152, 417-435. [CrossRef]

32. Marichal, J.L. Entropy of discrete Choquet capacities. Eur. J. Oper. Res. 2002, 137, 612-624. [CrossRef]

33. Wu, J.Z.; Zhang, Q. 2-order additive fuzzy measure identification method based on diamond pairwise comparison and maximum entropy principle. Fuzzy Optim. Decis. Mak. 2010, 9, 435-453. [CrossRef]

34. Saaty, T. The Modern Science of Multicriteria Decision Making and Its Practical Applications: The AHP/ANP Approach. Oper. Res. 2013, 61. [CrossRef]

35. Lee, S.H. Applying system dynamics to strategic decision making in construction. Front. Eng. Manag. 2017, 4, 35-40. [CrossRef]

36. Chateauneuf, A.; Jaffray, J.Y. Some characterizations of lower probabilities and other monotone capacities through the use of Möbius inversion. Math. Soc. Sci. 1989, 17, 263-283. [CrossRef]

37. Shapley, L.S. A value for n-person games. Contrib. Theory Games 1953, 2, 307-317.

38. Banzhaf III, J.F. Weighted voting doesn't work: A mathematical analysis. Rutgers L. Rev. 1964, $19,317$.

39. Wang, Z.; Klir, G.J. Generalized Measure Theory; Springer Science \& Business Media: New York, NY, USA, 2010.

40. Kolesárová, A.; Li, J.; Mesiar, R. k-additive aggregation functions and their characterization. Eur. J. Oper. Res. 2018, 265, 985-992. [CrossRef]

41. Wu, J.Z.; Beliakov, G. Nonadditivity index and capacity identification method in the context of multicriteria decision making. Inf. Sci. 2018, 467, 398-406. [CrossRef]

(c) 2019 by the authors. Licensee MDPI, Basel, Switzerland. This article is an open access article distributed under the terms and conditions of the Creative Commons Attribution (CC BY) license (http:/ / creativecommons.org/licenses/by/4.0/). 